\title{
GENERALIZED RESOLVENTS AND SPECTRUM FOR A CERTAIN CLASS OF PERTURBED SYMMETRIC OPERATORS
}

\section{A. HEBBECHE}

Received 3 February 2004 and in revised form 20 May 2004

The generalized resolvents for a certain class of perturbed symmetric operators with equal and finite deficiency indices are investigated. Using the Weinstein-Aronszajn formula, we give a classification of the spectrum.

\section{Introduction}

The present paper is concerned with the study of spectral properties for a certain class of linear symmetric operator $T$, defined in the Hilbert space $H$ of the form $T=A+$ $B$, where $A$ is a closed linear symmetric operator, with nondensely defined domain in general, $D(A) \subset H$, and $B$ is a finite-rank operator of the form

$$
B f=\sum_{k=1}^{n} a_{k}\left(f, y_{k}\right) y_{k},
$$

where $y_{1}, y_{2}, \ldots, y_{n}$ is a linearly independant system in $H, a_{1}, a_{2}, \ldots, a_{n} \in \mathbb{R}$. We remark that the operator $T$ can be considered as a perturbation of the operator $A$ by the finiterank operator $B$.

The case when $A$ is a first-order or second-order differential operator in the spaces $L^{2}(0,2 \pi), L^{2}(0, \infty)$ or in the Hilbert space of vector-valued functions, and $B$ is a onedimensional perturbation $(n=1)$, has been studied by many authors (see, e.g., $[9,20$, 24]).

In particular, certain integrodifferential equations of the above type occur in quantum mechanical scattering theory [8].

In this paper, the generalized resolvents of perturbed symmetric operator $T$ with equal and finite deficiency indices are investigated. Using the Weinstein-Aronszajn formula (see, e.g., [18]), we give a classification of the spectrum. Finally, the obtained results are applied to the study of two classes of first-order and second-order differential operators.

We note that the spectral theory of perturbed symmetric and selfadjoint operators have been investigated using various methods by many authors $[3,4,5,6,11,12,13,14$, $15,16,17,21,22]$. 


\section{Preliminaries}

Let $A$ be a closed symmetric operator with nondensely defined domain in a separable Hilbert space $H$ with equal deficiency indices $(m, m)$, and $m<\infty$. We denote by $\rho(A)$ the resolvent set of the operator $A$, the resolvent operator $R_{\lambda}(A)$ of $A$ is defined as $R_{\lambda}(A)=$ $(A-\lambda I)^{-1}$. The complement of $\rho(A)$ in the complex plane is called the spectrum of $A$ and denoted by $\sigma(A)$. There is a decomposition of the spectrum $\sigma(A)$ into three disjoint subsets, at least one of which is not empty $[1,2,10]$ :

$$
\sigma(A)=P \sigma(A) \cup C \sigma(A) \cup P C \sigma(A),
$$

$\operatorname{P\sigma }(A)$ is called the point spectrum, $C \sigma(A)$ the continuous spectrum, and $\operatorname{PC} \sigma(A)$ the point-continuous spectrum. We denote the essential spectrum of the operator $A$ by $\sigma_{e}(A)$ $=C \sigma(A) \cup P C \sigma(A)$.

For arbitrary $\lambda \in \mathbb{C}$, we denote $P_{\lambda}=N_{\lambda} \cap\left(D(A) \oplus N_{\bar{\lambda}}\right)$, where $N_{\lambda}=H \Theta(A-\lambda I) D(A)$ is the deficiency subspace of the operator $A[1,2]$.

It is known [23] that $P_{\lambda}=\{0\}$ if and only if $\overline{D(A)}=H$, and if $\overline{D(A)} \neq H$, then the subset

$$
G_{\lambda}=\left\{[\varphi, \psi] \in N_{\lambda} \times N_{\bar{\lambda}}: \varphi-\psi \in D(A)\right\}
$$

is a graph of the isometric operator $X_{\lambda}$ with domain $P_{\lambda}$ and values in $P_{\bar{\lambda}}$.

We denote by $\mathfrak{J}$ the set of linear operators $F$ defined from $N_{i}$ to $N_{-i}$, such that $\|F\| \leq$ 1. For each analytic operator-valued function $F(\lambda)$ in $\mathbb{C}^{+}$, with $\mathbb{C}^{+}=\{\lambda: \operatorname{Im} \lambda>0\}$, and values in $\mathfrak{I}$, we introduce the set $\Omega_{F}(\infty)$ consisting of elements $h \in N_{i}$ such that

$$
\lim _{\lambda \rightarrow \infty, \lambda \in \mathbb{C}_{\varepsilon}^{+}}|\lambda|[\|h\|-\|F(\lambda) h\|]<\infty,
$$

where $C_{\varepsilon}^{+}=\left\{\lambda \in \mathbb{C}^{+}: \varepsilon<\arg \lambda<\pi-\varepsilon\right\}, 0<\varepsilon<\pi / 2$.

It is known [27] that $\Omega_{F}(\infty)$ is a vector space and for each $h \in \Omega_{F}(\infty)$,

$$
\lim _{\lambda \rightarrow \infty, \lambda \in \mathbb{C}_{\varepsilon}^{+}} F(\lambda) h=F_{0}(\infty) h
$$

exists in the sense of the strong topology, and $F_{0}(\infty)$ is an isometric operator.

According to the theory of Štraus [28], the generalized resolvents of $A$ are given by the formula

$$
R_{\lambda}(A)=R_{\lambda}=\left(A_{F(\lambda)}-\lambda I\right)^{-1}, \quad R_{\bar{\lambda}}=R_{\lambda}^{*}, \quad \lambda \in \mathbb{C}^{+},
$$

where $A_{F(\lambda)}$ is an extension of $A$ which is determined by the function $F(\lambda)$, whose values are operators from the deficiency subspace $N_{i}$ to the deficiency subspace $N_{-i}$ such that $\|F(\lambda)\| \leq 1$ and $F(\lambda)$ satisfy the condition

$$
F_{0}(\infty) \psi=X_{i} \psi, \quad \text { for } \psi=0 \text { only, }
$$


then $A_{F(\lambda)}$ is a restriction on $H$ of a selfadjoint operator defined in a certain extended Hilbert space and is called quasiselfadjoint extension of the operator $A$ [28] defined on $D\left(A_{F(\lambda)}\right)=D(A)+(F(\lambda)-I) N_{i}$ by

$$
A_{F(\lambda)}(f+F(\lambda) \varphi-\varphi)=A f+i F(\lambda) \varphi+i \varphi, \quad f \in D(A), \varphi \in N_{i} .
$$

For selfadjoint extensions with exit in the space in which acts the considered operators, see, for example, $[12,21]$ and the references therein.

We denote by $\boldsymbol{N}$ the set of analytic operator functions $F(\lambda)$ in $\mathbb{C}^{+}$with values in $\mathfrak{J}$ satisfying the condition (2.6).

Remark 2.1. To each selfadjoint extension of the operator $A$ corresponds a certain constant operator function $F(\lambda)=V$, where $V$ is an isometric operator defined from $N_{i}$ over $N_{-i}$ satisfying the condition $V \psi=X_{i} \psi$ for $\psi=0$ only, and reciprocally.

We denote by $\AA$ a selfadjoint extension of $A$ and we introduce the operator

$$
\stackrel{\circ}{U}_{\lambda \lambda_{0}}=\left(\stackrel{\circ}{A}-\lambda_{0} I\right)(\stackrel{\AA}{A}-\lambda I)^{-1}, \quad \operatorname{Im} \lambda>0 .
$$

We note that (see [19])

$$
\stackrel{\circ}{U}_{\lambda \lambda_{0}} N_{\overline{\lambda_{0}}}=N_{\bar{\lambda},}, \quad(\operatorname{Im} \lambda)\left(\operatorname{Im} \lambda_{0}\right) \neq 0
$$

We denote by

$$
\varphi_{i}^{(1)}, \varphi_{i}^{(2)}, \ldots, \varphi_{i}^{(m)}
$$

a basis of $N_{-i}$. From (2.9), $\varphi_{\lambda}^{(k)}=\stackrel{\circ}{U}_{\lambda i} \varphi_{i}^{(k)}, k=1,2, \ldots, m$ form a basis for $N_{\bar{\lambda}}$. In particular, the vectors

$$
\varphi_{-i}^{(k)}=\stackrel{\circ}{U} \varphi_{i}^{(k)}, \quad k=1,2, \ldots, m
$$

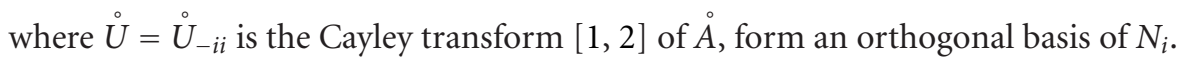

To get a convenient formula of the generalized resolvents of $A$, we will need the following notation:

$$
\Phi_{\lambda \mu}=(\lambda-\bar{\mu})\left[\left(\varphi_{\lambda}^{(k)}, \varphi_{\mu}^{(s)}\right)\right]_{k, s=1}^{m}, \quad C(\lambda)=\Phi_{\lambda i}^{-1} \Phi_{\lambda(-i)},
$$

where $E$ is the identity matrix of order $m, \Omega(\lambda)$ is an analytic matrix function in $\mathbb{C}^{+}$ corresponding, in the bases (2.10) and (2.11), to the operator function $F(\lambda) \in \mathcal{N}$ and $\varphi_{\lambda}=$ $\left(\varphi_{\lambda}^{(1)}, \ldots, \varphi_{\lambda}^{(m)}\right)^{t},\left(f, \varphi_{\bar{\lambda}}\right)^{t}=\left(\left(f, \varphi_{\bar{\lambda}}^{(1)}\right), \ldots,\left(f, \varphi_{\bar{\lambda}}^{(m)}\right)\right), t$ denotes the transpose, and $\left(\varphi_{\lambda}, g\right)$ is defined analogously. 
In what follows, we denote by $\Phi$ the set of matrices $\Omega(\lambda), \lambda \in \mathbb{C}^{+}$, associated in the bases (2.10) and (2.11) to the operator functions $F(\lambda) \in \kappa$.

According to the notation used in [7], the generalized resolvents of $A$ are given by

$$
\begin{gathered}
R_{\lambda}(A) f=R_{\lambda} f=\stackrel{\circ}{R}_{\lambda} f+\left(f, \varphi_{\bar{\lambda}}\right)^{t}[E-\Omega(\lambda)][C(\lambda) \Omega(\lambda)-E]^{-1} \Phi_{\lambda i}^{-1} \varphi_{\lambda}, \\
R_{\bar{\lambda}}=R_{\lambda}^{*}, \quad \lambda \in \mathbb{C}^{+},
\end{gathered}
$$

where $\stackrel{\circ}{R}_{\lambda}$ is the resolvent of $\AA$ and $\Omega(\lambda) \in \Phi$.

Remark 2.2. The formula (2.13) defines a resolvent of a selfadjoint extension of $A$ if and only if $\Omega(\lambda)$ is a unitary constant matrix.

\section{Resolvent and spectrum of a symmetric perturbed operator}

Let $T=A+B$ be defined on $D(T)=D(A)$, where $A$ is a linear closed symmetric operator in $H$ and $B$ is a finite-rank operator.

Lemma 3.1. For $\lambda \in \rho(A) \cap \rho(T)$, the resolvent $R_{\lambda}(T)$ of the operator $T$ is given by

$$
R_{\lambda}(T)=R_{\lambda}(A)-R_{\lambda}(A)\left[I+B R_{\lambda}(A)\right]^{-1} B R_{\lambda}(A) .
$$

Proof. For $\lambda \in \rho(A) \cap \rho(T)$, the operator

$$
R_{\lambda}(A)\left[I+B R_{\lambda}(A)\right]^{-1}=R_{\lambda}(T)
$$

exists and is bounded. Then, we get

$$
\begin{aligned}
(T-\lambda I)\left[R_{\lambda}(A)-R_{\lambda}(A)\left(I+B R_{\lambda}(A)\right)^{-1} B R_{\lambda}(A)\right] \\
=(A-\lambda I+B)\left[R_{\lambda}(A)-R_{\lambda}(A)\left(I+B R_{\lambda}(A)\right)^{-1} B R_{\lambda}(A)\right] \\
\quad=I+B R_{\lambda}(A)-\left(I+B R_{\lambda}(A)\right)\left(I+B R_{\lambda}(A)\right)^{-1} B R_{\lambda}(A)=I
\end{aligned}
$$

as required.

Remark 3.2. If $\left\|B R_{\lambda}(A)\right\|<1$, then from (3.1), we obtain

$$
R_{\lambda}(T)=R_{\lambda}(A)\left(I+B R_{\lambda}(A)\right)^{-1}=R_{\lambda}(A) \sum_{k=0}^{\infty}(-1)^{k}\left[B R_{\lambda}(A)\right]^{k} .
$$

Now, the aim is to give a convenient expression of $\left(I+B R_{\lambda}(A)\right)^{-1}$ in a more specific case.

So, we study in detail the case when $B$ is a finite-rank operator. Then,

$$
B f=\sum_{k=1}^{n} a_{k}\left(f, y_{k}\right) y_{k}, \quad f \in H,
$$

where $a_{1}, a_{2}, \ldots, a_{n} \in \mathbb{R} ;\left\{y_{1}, y_{2}, \ldots, y_{n}\right\}$ is a linearly independent system in $H$. If we put

$$
\left(I+B R_{\lambda}(A)\right)^{-1} B R_{\lambda}(A) f=y,
$$


we have

$$
y=B R_{\lambda}(A) f-B R_{\lambda}(A) y
$$

then, $y \in \operatorname{Im} B$, so that

$$
y=\sum_{k=1}^{n} c_{k} y_{k}
$$

From (3.7) and (3.8), we get

$$
\sum_{k=1}^{n} c_{k} y_{k}=B R_{\lambda}(A) f-\sum_{k=1}^{n} c_{k} B R_{\lambda}(A) y_{k},
$$

with

$$
c_{k}+a_{k} \sum_{j=1}^{n} c_{j}\left(R_{\lambda}(A) y_{j}, y_{k}\right)=a_{k}\left(R_{\lambda}(A) f, y_{k}\right) .
$$

The determinant $\Delta(\lambda)$ of the system (3.10) is given by

$$
\Delta(\lambda)=\operatorname{det}\left\{\left[\delta_{k j}+a_{k}\left(R_{\lambda}(A) y_{j}, y_{k}\right)\right]_{k, j=1}^{n}\right\}
$$

where $\delta_{k j}$ is the Kronecker symbol. If we suppose that $\Delta(\lambda) \neq 0$, the solution of (3.10) is given by

$$
c_{k}=c_{k}(\lambda ; f)=\frac{\left(f, \Delta_{k}(\lambda)\right)}{\Delta(\lambda)}, \quad k=1,2, \ldots, n
$$

where $\Delta_{k}(\lambda)$ is the determinant obtained from $\overline{\Delta(\lambda)}$ by replacing the $k$ th column by $\left[a_{j} R_{\bar{\lambda}}(A) y_{j}\right]_{j=1}^{n}$. So, from (3.1), we have

$$
R_{\lambda}(T) f=R_{\lambda}(A) f-\sum_{k=1}^{n} \frac{\left(f, \Delta_{k}(\lambda)\right)}{\Delta(\lambda)} R_{\lambda}(A) y_{k} .
$$

This completes the proof of the following theorem.

Theorem 3.3. Let $\lambda \in \rho(A)$ such that $\Delta(\lambda) \neq 0$. Then, $\lambda \in \rho(T)$ and the resolvent of the operator $T$ is given by (3.13).

Remark 3.4. From (3.13), we note that the resolvent $R_{\lambda}(T)$ is a perturbation of $R_{\lambda}(A)$ by a finite-rank operator. 
Remark 3.5. For the particular case $n=1$ and $a_{1}=1$, the formula (3.13) was established in [9].

Remark 3.6. If $\lambda \in \rho(A)$ such that $\Delta(\lambda)=0$, then $\lambda$ is an eigenvalue of the operator $T$.

Proof. We can show that there exists an element

$$
\psi=\sum_{k=1}^{n} \alpha_{k} y_{k}
$$

such that $R_{\lambda}(A) \psi$ is an eigenvector of the operator $T$, corresponding to the eigenvalue $\lambda$. Consequently, we have

$$
a_{k} \sum_{j=1}^{n} \alpha_{j}\left(R_{\lambda}(A) y_{j}, y_{k}\right)+\alpha_{k}=0, \quad k=\overline{1, n} .
$$

Since the determinant of this system $\Delta(\lambda)=0$, it admits a nontrivial solution, which gives the desired result.

Theorem 3.7. Let $\mu$ be a fixed complex number. Then, the following holds.

(a) If $\mu \in \rho(A)$ and $\Delta(\mu) \neq 0$, then $\mu \in \rho(T)$.

(b) If $\mu \in \rho(A)$ and $\Delta(\mu)=0$, then $\mu \in P \sigma(T)$ and the multiplicity of $\mu$ as an eigenvalue of $T$ is equal to the order of the zero of $\Delta(\lambda)$ at $\mu$.

(c) If $\mu \in P \sigma(A)$ and $\mu$ of multiplicity $k>0$ and if $\mu$ is a pole of $\Delta(\lambda)$ of multiplicity $p$ $(k \geq p)$, then

(1) for $k>p$, it holds that $\mu \in P \sigma(T)$ of multiplicity $(k-p)$,

(2) for $k=p$, it holds that $\mu \in \rho(T)$.

(d) If $\mu \in \operatorname{Po}(A)$ is neither a zero, nor a pole of $\Delta(\lambda)$, then $\mu \in P \sigma(T)$.

(e) If $\mu \in P \sigma(A)$ of multiplicity $k$ and $\mu$ is a root of the function $\Delta(\lambda)$ of order $p$, then $\mu \in P \sigma(T)$ of order $(k+p)$.

(f) The essential spectra $\sigma_{e}(A)$ and $\sigma_{e}(T)$, respectively of the operators $A$ and $T$, coincide.

Proof. It is sufficient to evaluate the function

$$
C(\lambda)=\operatorname{det}\left\{I+B R_{\lambda}(A)\right\}
$$

To this end, let $y \in \operatorname{Im} B$. Then,

$$
B R_{\lambda}(A) y=\sum_{k=1}^{n} a_{k}\left(y, R_{\lambda}^{*}(A) y_{k}\right) y_{k}
$$

it is clear that $C(\lambda)=\Delta(\lambda)$, and the function $\Delta(\lambda)$ is meromorphic in $\rho(A) \cup P \sigma(A)$. From the formula of Weinstein and Aronszajn [18], we have

$$
\bar{\vartheta}(\lambda ; T)=\bar{\vartheta}(\lambda ; A)+\vartheta(\lambda ; \Delta),
$$


where

$$
\begin{aligned}
& \bar{\vartheta}(\lambda ; A)= \begin{cases}0 & \text { if } \lambda \in \rho(A), \\
k & \text { if } \lambda \in P \sigma(A) \text { and of multiplicity } k, \\
+\infty & \text { otherwise, }\end{cases} \\
& \vartheta(\lambda ; \Delta)= \begin{cases}k & \text { if } \lambda \text { is a zero of } \Delta(\lambda) \text { of order } k, \\
-k & \text { if } \lambda \text { is a pole of } \Delta(\lambda) \text { of order } k, \\
0 & \text { for other } \lambda \in \Omega,\end{cases}
\end{aligned}
$$

which gives the desired result.

\section{Generalized resolvents}

Now, we suppose that $A$ is a symmetric operator with deficiency indices $(m, m), m<\infty$.

Lemma 4.1. Let $\lambda \in \mathbb{C}$ such that $\operatorname{Im} \lambda>0$ and $\varphi_{\lambda}(A) \in N_{\bar{\lambda}}(A)$. Then, the element $\varphi_{\lambda}(T)$, defined by the formula

$$
\varphi_{\lambda}(T)=D(\lambda) \varphi_{\lambda}(A)=\varphi_{\lambda}(A)-\sum_{k=1}^{n} \frac{\left(\varphi_{\lambda}(A), \stackrel{\circ}{g}_{k}(\lambda)\right)}{\stackrel{\circ}{\Delta}(\lambda)} R_{\lambda}(\AA) y_{k},
$$

is an element of the deficiency subspace $N_{\bar{\lambda}}(T)$, where

$$
D(\lambda)=I-R_{\lambda}\left(\stackrel{\circ}{\AA}\left[I+B R_{\lambda}(\stackrel{\circ}{\AA})\right]^{-1} B=I-R_{\lambda}(\stackrel{\circ}{T}) B, \quad \stackrel{\circ}{g}_{k}(\lambda)=(\stackrel{\circ}{A}-\bar{\lambda} I) \stackrel{\circ}{\Delta}_{k}(\lambda),\right.
$$

$\AA_{\Delta}(\lambda)$ and $\AA_{k}(\lambda)$ are defined similarly as $\Delta(\lambda)$ and $\Delta_{k}(\lambda)$ in the formula (3.13) by putting the operator $\AA$ instead of the operator $A$.

Proof. Since the operators $\AA$ and $\stackrel{\circ}{T}=\stackrel{A}{ }+B$ are selfadjoint and $\lambda$ is nonreal, then $\lambda \in$ $\rho(\stackrel{\circ}{A}) \cap \rho(\stackrel{\circ}{T})$. In addition, from Theorem 3.3 we have $\stackrel{\circ}{\Delta}(\lambda) \neq 0$. Furthermore, for each $f \in D(A)=D(T)$, we have

$$
\begin{aligned}
\left([\stackrel{\circ}{T}-\bar{\lambda} I] f, D(\lambda) \varphi_{\lambda}(A)\right) & =\left(D^{*}(\lambda)[\stackrel{\circ}{T}-\bar{\lambda} I] f, \varphi_{\lambda}(A)\right) \\
& =\left(\left[I-B R_{\bar{\lambda}}(\stackrel{\circ}{T})\right](\stackrel{\circ}{T}-\bar{\lambda} I) f, \varphi_{\lambda}(A)\right) \\
& =\left((\stackrel{\AA}{\AA}-\bar{\lambda} I) f, \varphi_{\lambda}(A)\right) \\
& =0,
\end{aligned}
$$

and the equality

$$
\varphi_{\lambda}(T)=\varphi_{\lambda}(A)-\sum_{k=1}^{n} \frac{\left(\varphi_{\lambda}(A), \stackrel{\circ}{g}_{k}(\lambda)\right)}{\stackrel{\circ}{\Delta}(\lambda)} R_{\lambda}(\AA) y_{k}
$$

results from (3.13).

Remark 4.2. We note that if $\varphi_{\lambda}(A) \neq 0$, then $\varphi_{\lambda}(T) \neq 0$. 
Proof. If we suppose the contrary, we obtain $R_{\lambda}(\stackrel{\circ}{T}) B \varphi_{\lambda}(A)=\varphi_{\lambda}(A)$, which gives $\AA^{\circ} \varphi_{\lambda}(A)=$ $\lambda \varphi_{\lambda}(A)$. This leads to a contradiction, since a selfadjoint operator can not have nonreal eigenvalues.

Remark 4.3. If $D(A)$ is dense in $H$, then $\varphi_{\lambda}(A)$ and $\varphi_{\lambda}(T)$ are, respectively, eigenfunctions of the operators $A^{*}$ and $T^{*}$, corresponding to the eigenvalues $\bar{\lambda}$.

Let $\varphi_{i}^{(k)}(T)=D(i) \varphi_{\lambda}^{(k)}(A), k=1,2, \ldots, m$, defined by the formula (4.1). If $\varphi_{i}^{(1)}(A)$, $\varphi_{i}^{(2)}(A), \ldots, \varphi_{i}^{(m)}(A)$ is a basis of the deficiency subspace $N_{i}(A)$ of the operator $A$, then $\varphi_{i}^{(1)}(T), \varphi_{i}^{(2)}(T), \ldots, \varphi_{i}^{(m)}(T)$ is a basis of the deficiency subspace $N_{i}(T)$ of the operator $T$. Putting

$$
\begin{gathered}
\stackrel{\circ}{U}_{\lambda \lambda_{0}}(\stackrel{\circ}{T})=\left(\stackrel{\circ}{T}-\lambda_{0} I\right) R_{\lambda}(\stackrel{\circ}{T}), \quad \varphi_{\lambda}^{(k)}(T)=\stackrel{\circ}{U}_{\lambda i}(\stackrel{\circ}{T}) \varphi_{i}^{(k)}(T), \quad k=1,2, \ldots, m, \\
\varphi_{\lambda}(T)=\left(\varphi_{\lambda}^{(1)}(T), \ldots, \varphi_{\lambda}^{(m)}(T)\right)^{t}, \quad \Phi_{\lambda \mu}(T)=(\lambda-\bar{\mu})\left[\left(\varphi_{\lambda}^{(k)}(T), \varphi_{\mu}^{(j)}(T)\right)\right]_{k, j=1}^{m},
\end{gathered}
$$

$C(\lambda)=\Phi_{\lambda i}^{-1}(T) \Phi_{\lambda(-i)}(T)$ denotes the characteristic matrix of the operator $T$, and $\omega(\lambda)$ the corresponding matrix of order $m \times m$, in the bases $\varphi_{i}^{(1)}(T), \varphi_{i}^{(2)}(T), \ldots, \varphi_{i}^{(m)}(T)$ and $\varphi_{-i}^{(1)}(T), \varphi_{-i}^{(2)}(T), \ldots, \varphi_{-i}^{(m)}(T)$.

THEOREM 4.4. The set of all generalized resolvents of the operator $T$ is given by

$$
R_{\lambda}(T) f=R_{\lambda}(\stackrel{\circ}{T}) f+\left(f, \varphi_{\bar{\lambda}}(T)\right)^{t}[E-\omega(\lambda)][C(\lambda) \omega(\lambda)-E]^{-1} \Phi_{\lambda i}^{-1}(T) \varphi_{\lambda}(T), \quad \forall f \in H,
$$

where

$$
R_{\lambda}(\stackrel{\circ}{T}) f=R_{\lambda}(\stackrel{\circ}{\AA}) f-\sum_{k=1}^{n} \frac{\left(f, \stackrel{\circ}{\Delta}_{k}(\lambda)\right)}{\stackrel{\circ}{\Delta}(\lambda)} R_{\lambda}(\stackrel{\circ}{A}) y_{k}
$$

Proof. The proof results from Lemma 4.1 and formula (2.13).

We denote, respectively, by $A_{\omega}$ and $T_{\omega}$ the quasiselfadjoint extensions of operators $A$ and $T$ corresponding to the operator function $F(\lambda) \in \mathfrak{J}$, defined by the matrix $\omega(\lambda)$.

Remark 4.5. To selfadjoint extensions of these operators correspond the constant unitary matrices $\omega=\left[\omega_{i j}\right]$.

Theorem 4.6. Suppose that $y_{1}, y_{2}, \ldots, y_{n} \in \operatorname{Im} A, \mu$ is an eigenvalue of the quasiselfadjoint extension $A_{\omega}$ of the operator $A, \mu \in P \sigma\left(A_{\omega}\right)$. If $\mu \in \rho(\AA)$ and $\stackrel{\circ}{\Delta}(\mu) \neq 0$, then $\mu$ is an eigenvalue of the operator $T_{\omega}=A_{\omega}+B$ and the corresponding eigenfunction $\varphi_{\mu}\left(T_{\omega}\right)$ is given by

$$
\varphi_{\mu}\left(T_{\omega}\right)=D(\mu) \varphi_{\mu}\left(A_{\omega}\right)=\varphi_{\mu}\left(A_{\omega}\right)-\sum_{k=1}^{n} \frac{\left(\varphi_{\mu}\left(A_{\omega}\right), \stackrel{\circ}{g}_{k}(\mu)\right)}{\stackrel{\circ}{\Delta}(\mu)} R_{\mu}(\AA) y_{k},
$$

where $\varphi_{\mu}\left(A_{\omega}\right)$ is the eigenfunction of the operator $A_{\omega}$, corresponding to the eigenvalue $\mu$. 
Proof. Since $y_{1}, y_{2}, \ldots, y_{n} \in \operatorname{Im} A$, then $B \varphi_{\mu}(A) \in \operatorname{Im} A$. We also have

$$
\varphi_{\mu}\left(T_{\omega}\right)=D(\mu) \varphi_{\mu}\left(A_{\omega}\right)=\varphi_{\mu}\left(A_{\omega}\right)-R_{\mu}(\stackrel{\circ}{T}) B \varphi_{\mu}(A)=\varphi_{\mu}\left(A_{\omega}\right)-\psi_{\mu},
$$

where

$$
\psi_{\mu}=R_{\mu}(\stackrel{\circ}{T}) B \varphi_{\mu}(A) \in D(A) .
$$

Then,

$$
\begin{aligned}
T_{\omega} \varphi_{\mu}\left(T_{\omega}\right) & =T_{\omega}\left(\varphi_{\mu}\left(A_{\omega}\right)-\psi_{\mu}\right) \\
& =\left(A_{\omega}+B\right) \varphi_{\mu}\left(A_{\omega}\right)-T_{\omega} R_{\mu}(\stackrel{\circ}{T}) B \varphi_{\mu}(A) \\
& =\mu \varphi_{\mu}\left(A_{\omega}\right)+B \varphi_{\mu}\left(A_{\omega}\right)-B \varphi_{\mu}\left(A_{\omega}\right)+\mu R_{\mu}(\stackrel{\circ}{T}) B \varphi_{\mu}(A) \\
& =\mu \varphi_{\mu}\left(T_{\omega}\right) .
\end{aligned}
$$

\section{Applications}

5.1. Perturbed first-order differential operator. Consider in $L^{2}(0,2 \pi)$ the operator $T=$ $A+B$, where $A$ is defined by $A y=i y^{\prime}$ with domain $D(A)=H_{0}^{1}(0,2 \pi)$ and $B$ is given by

$$
(B y)(x)=\sum_{k=1}^{n} a_{k}\left(y, y_{k}\right) y_{k}(x),
$$

where $y_{1}, y_{2}, \ldots, y_{n} \in L^{2}(0,2 \pi)$ and $a_{k} \in \mathbb{R}$, for all $k=\overline{1, n}$. From $[1,2]$, the operator $A$ is regular symmetric of deficiency indices $(1,1)$ and each selfadjoint extension of $A$ has a discrete spectrum.

THEOREM 5.1. The generalized resolvent $R_{\lambda}\left(T_{\theta}\right)$ of $T$, corresponding to the function $\omega(\lambda)=$ $\theta(\lambda)$, is an integral operator with kernel

$$
K(x, t)=\left[1_{[x, 2 \pi]}(x)+\frac{1}{\theta(\lambda) e^{2 \pi \lambda i}+1}\right] e^{i \lambda(t-x)}+\sum_{k=1}^{n} \theta_{k}(\lambda, x) \phi_{k}(\lambda, t),
$$

where $1_{[x, 2 \pi]}(x)$ is the characteristic function of the interval $[x, 2 \pi]$,

$$
\phi_{k}(\lambda, t)=\left(\Delta_{k}^{\theta}(\lambda)\right)(t), \quad \theta_{k}(\lambda, x)=\frac{\left(R_{\lambda}\left(A_{\theta}\right) y_{k}\right)(x)}{\Delta^{\theta}(\lambda)},
$$

where $R_{\lambda}\left(A_{\theta}\right)$, associated to the function $\theta(\lambda)$, is given by

$$
\left(R_{\lambda}\left(A_{\theta}\right) y\right)(x)=\int_{0}^{x} y(t) e^{i \lambda(t-x)} d t-\frac{1}{\theta(\lambda) e^{2 \pi t i}+1} \int_{0}^{2 \pi} y(t) e^{\lambda i(t-x)} d t
$$

with

$$
\Delta^{\theta}(\lambda)=\left\{\delta_{k_{j}}+a_{k}\left(R_{\lambda}\left(A_{\theta}\right) y_{j}, y_{k}\right)\right\}
$$

and $\Delta_{k}^{\theta}$ is the determinant obtained from $\overline{\Delta^{\theta}(\lambda)}$ replacing the kth column by $\left[a_{k} R_{\bar{\lambda}}\left(A_{\theta}\right) y_{k}\right]_{1}^{n}$. 
Proof. The proof results from [26] and Theorem 3.3.

Corollary 5.2. Let $T_{\theta}$ be a selfadjoint extension of $T$ corresponding to the function $\theta$, $|\theta|=1$.

(1) The spectrum of $T_{\theta}$ is simple if and only if the roots of $\Delta^{\theta}(\lambda)$ are simple and for $k=0, \pm 1, \pm 2, \ldots, \Delta^{\theta}\left(1 / 2+k-\varphi_{0} / 2 \pi\right) \neq 0$, where $\left\{1 / 2+k-\varphi_{0} / 2 \pi\right\}$ is the spectrum of $A_{\theta}$, and $\varphi_{0}=\arg \theta$.

(2) $\sigma\left(T_{\theta}\right)=P \sigma\left(T_{\theta}\right)=E_{1} \cup E_{2}$, where $E_{1}$ is the set of points of $\sigma\left(A_{\theta}\right)=\left\{1 / 2+k-\varphi_{0} / 2 \pi\right.$, $k=0, \pm 1, \pm 2, \ldots\}$ in which $\Delta^{\theta}(\lambda)$ is analytic, $E_{2}$ is the set of roots of $\Delta^{\theta}(\lambda)$.

Proof. The proof results from (5.4), Theorem 3.7, and Lemma 4.1.

5.2. Perturbed second-order differential operator. Consider in $L^{2}(0, \infty)$ the operator $T=A+B$, where $A$ is defined by

$$
A y=-y^{\prime \prime}+x^{2} y
$$

with domain $D(A)$ consisting of all variables $y$ which satisfy

(i) $y \in L^{2}(0, \infty)$,

(ii) $y^{\prime}$ is absolutely continuous on all compact subintervals of $[0, \infty[$,

(iii) $A y \in L^{2}(0, \infty)$,

(IV) $y(0)=y(\infty)=\lim _{x \rightarrow \infty} y(x)=0, y^{\prime}(0)=y^{\prime}(\infty)=0$,

and $B$ is given by

$$
(B y)(x)=\sum_{k=1}^{n} a_{k}\left(y, y_{k}\right) y_{k}(x)
$$

where $y_{1}, y_{2}, \ldots, y_{n} \in L^{2}(0,2 \pi)$ and $a_{k} \in I R$, for all $k=\overline{1, n}$.

From $[1,2]$, the operator $A$ is symmetric of deficiency indices $(1,1)$. Let $u_{1}, u_{2}$ be two solutions of (5.6), satisfying the initial conditions

$$
\begin{array}{ll}
u_{1}(0, \lambda)=1, & \left.u_{1}^{\prime}(x, \lambda)\right|_{x=0}=0, \\
u_{2}(0, \lambda)=0, & \left.u_{2}^{\prime}(x, \lambda)\right|_{x=0}=-1 .
\end{array}
$$

There exists a function $m(\lambda)[29]$ analytic in $\mathbb{C} \backslash \mathbb{R}$ such that

$$
\psi(x, \lambda)=u_{2}(x, \lambda)+m(\lambda) u_{1}(x, \lambda) \in L^{2}(0, \infty)
$$

TheOREM 5.3. The generalized resolvents $R_{\lambda}\left(T_{\theta}\right)$ of the operator $T$ are defined by

$$
R_{\lambda}\left(T_{\theta}\right) y=R_{\lambda}\left(A_{\theta}\right) y-\sum_{k=1}^{n} \frac{\left(y, \Delta_{k}^{\theta}(\lambda)\right)}{\Delta^{\theta}(\lambda)} R_{\lambda}\left(A_{\theta}\right) y_{k}, \quad \operatorname{Im} \lambda>0,
$$


where

$$
\begin{gathered}
R_{\lambda}\left(A_{\theta}\right) y=\psi(x, \lambda) \int_{0}^{x} y(s) u_{1}(s, \lambda) d s+u_{1}(x, \lambda) \int_{x}^{\infty} y(s) \psi(s, \lambda) d s \\
\quad-\frac{\psi(x, \lambda)}{\theta(\lambda)+m(\lambda)} \int_{0}^{\infty} y(s) \psi(s, \lambda) d s, \\
\Delta^{\theta}(\lambda)=\operatorname{det}\left\{\sigma_{j_{k}}+a_{k}\left(R_{\lambda}\left(A_{\theta}\right) y_{j}, y_{k}\right)\right\}, \quad \lambda \in \mathbb{C}^{+},
\end{gathered}
$$

with $\theta(\lambda)$ an arbitrary function analytic in $\mathbb{C}^{+}$and such that $\operatorname{Im} \theta(\lambda) \geq 0$ or $\theta(\lambda)$ is an infinite constant.

Proof. First, we show that for $\lambda \in \mathbb{C}^{+}, \Delta^{\theta}(\lambda) \neq 0$ (then, $\Delta^{\theta} \neq 0$ ). We know (see $[1,2]$ ) that for each quasiselfadjoint extension of a symmetric operator, $\mathbb{C}^{+}$is contained in the set of regular points of this operator. Then, if $\lambda \in \mathbb{C}^{+}$, we have $\lambda \in \rho\left(A_{\theta}\right)$ and $\lambda \in \rho\left(T_{\theta}\right)$. If we suppose that $\lambda \in \mathbb{C}^{+}$and $\Delta^{\theta}(\lambda)=0$, from Theorem 3.7, we obtain $\lambda \in P \sigma\left(T_{\theta}\right)$, which is a contradiction. The formula (5.11) results from [25]. Using Theorem 3.3, we end the proof.

Corollary 5.4. Let $T_{\theta}$ be a selfadjoint extension associated to $\theta \in \overline{I R}$, let $\lambda_{1}, \lambda_{2}, \ldots$ be the roots of $\Delta^{\theta}(\lambda)$ in $\rho\left(A_{\theta}\right)$ and let $z_{1}, z_{2}, \ldots$ be the poles of $\Delta^{\theta}(\lambda)$. Then,

$$
P \sigma\left(T_{\theta}\right)=\left(P \sigma\left(A_{\theta}\right) \backslash\left\{z_{i}\right\}_{1}^{\infty}\right) \cup\left\{\lambda_{j}\right\}_{1}^{\infty} .
$$

Proof. The proof results from (b) and (c) of Theorem 3.7.

\section{Acknowledgment}

The author is grateful to the editor and the anonymous referees for their valuable comments and helpful suggestions which have much improved the presentation of the paper.

\section{References}

[1] N. I. Akhiezer and I. M. Glazman, Theory of Linear Operators in Hilbert Space. Vol. I, Frederick Ungar, New York, 1961, translated from Russian by Merlynd Nestell.

[2] _ Theory of Linear Operators in Hilbert Space. Vol. II, Frederick Ungar, New York, 1963, translated from Russian by Merlynd Nestell.

[3] S. Albeverio, V. Koshmanenko, P. Kurasov, and L. Nizhnik, On approximations of rank one $\mathscr{H}_{-2}$ perturbations, Proc. Amer. Math. Soc. 131 (2003), no. 5, 1443-1452.

[4] S. Albeverio and P. Kurasov, Rank one perturbations, approximations, and selfadjoint extensions, J. Funct. Anal. 148 (1997), no. 1, 152-169.

[5] _ Rank one perturbations of not semibounded operators, Integral Equations Operator Theory 27 (1997), no. 4, 379-400.

[6] Singular Perturbations of Differential Operators, London Mathematical Society Lecture Note Series, vol. 271, Cambridge University Press, Cambridge, 2000.

[7] E. L. Aleksandrov, The resolvents of a symmetric nondensely defined operator, Izv. Vysš. Učebn. Zaved. Matematika 98 (1970), no. 7, 3-12.

[8] R. A. Buckingham and H. S. W. Massey, The scattering of neutrons by deuterons and the nature of nuclear forces, Proc. Roy. Soc. Ser. A 179 (1941), no. 977, 123-151.

[9] E. A. Catchpole, An integro-differential operator, J. London Math. Soc. (2) 6 (1973), 513-523. 
[10] J. C. Chaudhuri and W. N. Everitt, On the spectrum of ordinary second order differential operators, Proc. Roy. Soc. Edinburgh Sect. A 68 (1969), 95-119.

[11] V. Derkach, S. Hassi, and H. S. V. de Snoo, Operator models associated with singular perturbations, Methods Funct. Anal. Topology 7 (2001), no. 3, 1-21.

[12] Rank one perturbations in a Pontryagin space with one negative square, J. Funct. Anal. 188 (2002), no. 2, 317-349.

[13] Singular perturbations of self-adjoint operators, Math. Phys. Anal. Geom. 6 (2003), no. 4, 349-384.

[14] V. A. Derkach, S. Hassi, M. M. Malamud, and H. S. V. de Snoo, Generalized resolvents of symmetric operators and admissibility, Methods Funct. Anal. Topology 6 (2000), no. 3, 24-55.

[15] A. Dijksma, H. Langer, and H. S. V. de Snoo, Generalized coresolvents of standard isometric operators and generalized resolvents of standard symmetric relations in Krĕn spaces, Topics in Operator Theory: Ernst D. Hellinger Memorial Volume, Oper. Theory Adv. Appl., vol. 48, Birkhäuser, Basel, 1990, pp. 261-274.

[16] S. Hassi and H. S. V. de Snoo, On rank one perturbations of selfadjoint operators, Integral Equations Operator Theory 29 (1997), no. 3, 288-300.

[17] One-dimensional graph perturbations of selfadjoint relations, Ann. Acad. Sci. Fenn. Math. 22 (1997), no. 1, 123-164.

[18] T. Kato, Perturbation Theory for Linear Operators, Die Grundlehren der mathematischen Wissenschaften, vol. 132, Springer-Verlag, New York, 1966.

[19] M. G. Krein, The fundamental propositions of the theory of representations of Hermitian operators with deficiency index ( $m, m)$, Ukrainian Math. J. 1 (1949), no. 2, 3-66 (Russian).

[20] O. P. Kruglikova, Generalized resolvents and spectral functions of a first-order integro-differential operator in the space of vector-valued functions, Functional Analysis, vol. 36, Ulyanovsk. Gos. Ped. Univ., Ulyanovsk, 1997, pp. 24-30.

[21] P. Kurasov, $\mathscr{H}_{-n}$-perturbations of self-adjoint operators and Krein's resolvent formula, Integral Equations Operator Theory 45 (2003), no. 4, 437-460.

[22] M. M. Malamud, On a formula for the generalized resolvents of a non-densely defined Hermitian operator, Ukrainian Math. J. 44 (1992), no. 12, 1658-1688 (Russian), (English translation: Sov. Math., Plenum Publ. Corp., (1993), 1522-1546).

[23] M. A. Neumark, Self-adjoint extensions of the second kind of a symmetric operator, Bull. Acad. Sci. URSS. Sér. Math. 4 (1940), 53-104 (Russian).

[24] G. I. Sin'ko, On the spectral theory of a second-order integro-differential operator, Functional Analysis, vol. 27, Ulyanovsk. Gos. Ped. Inst., Ulyanovsk, 1987, pp. 172-181.

[25] A. V. Štraus, On spectral functions of differential operators, Izv. Akad. Nauk SSSR Ser. Mat. 19 (1955), 201-220 (Russian).

[26] Spectral functions of a differential operator, Uspehi Mat. Nauk 13 (1958), no. 6 (84), 185-191 (Russian).

[27] - One-parameter families of extensions of a symmetric operator, Izv. Akad. Nauk SSSR Ser. Mat. 30 (1966), 1325-1352 (Russian).

[28] _ Extensions, characteristic functions and generalized resolvents of symmetric operators, Dokl. Akad. Nauk SSSR 178 (1968), 790-792 (Russian).

[29] E. C. Titchmarsh, Eigenfunction Expansions Associated with Second-Order Differential Equations. Part I, The Clarendon Press, Oxford University Press, New York, 1962.

A. Hebbeche: Laboratoire Equations Differentielles, Departement de Mathematiques, Faculté des Sciences, Université Mentouri, 25000 Constantine, Algeria

E-mail address: hebbeche@wissal.dz 


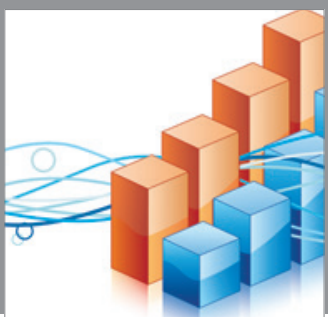

Advances in

Operations Research

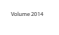

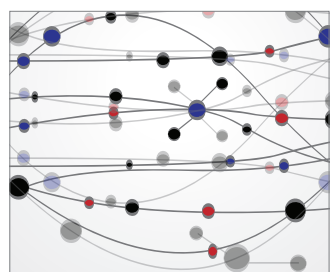

\section{The Scientific} World Journal
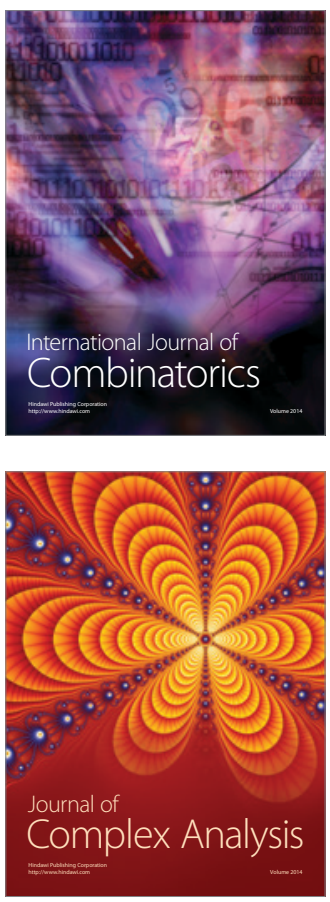

International Journal of

Mathematics and

Mathematical

Sciences
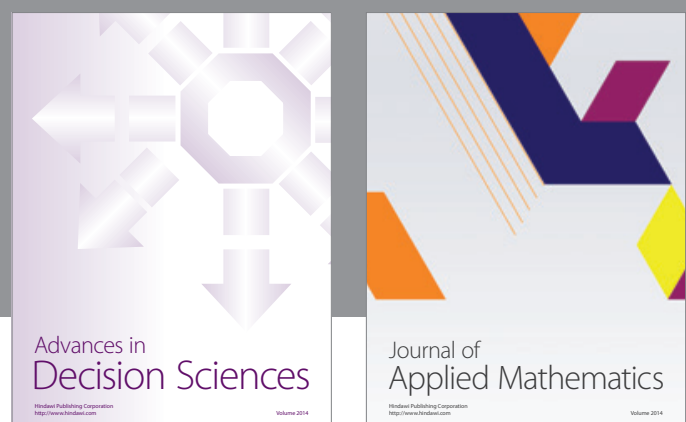

Journal of

Applied Mathematics
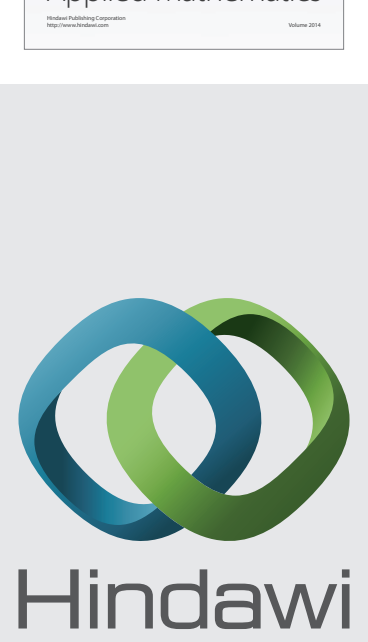

Submit your manuscripts at http://www.hindawi.com
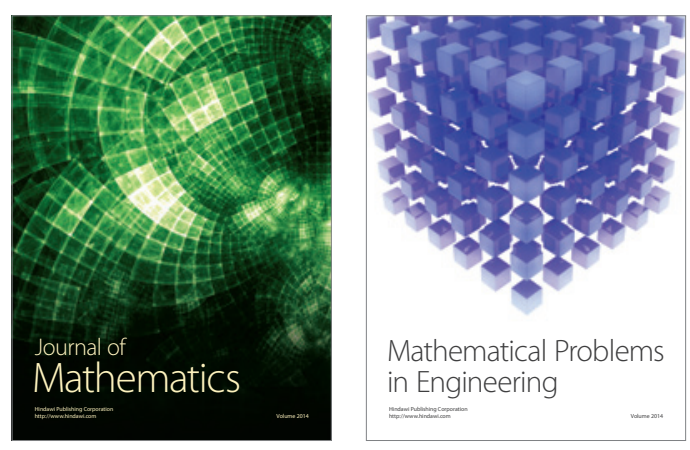

Mathematical Problems in Engineering
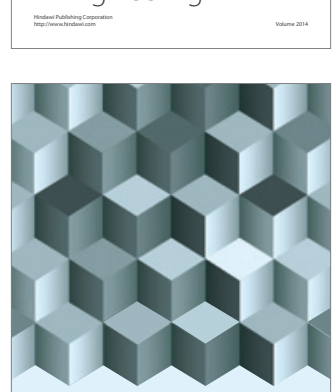

Journal of

Function Spaces
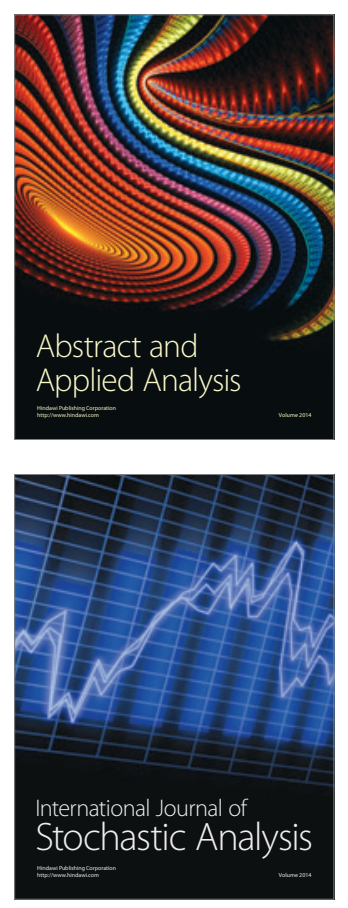

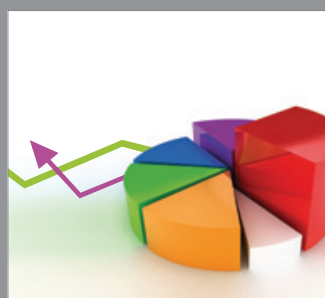

ournal of

Probability and Statistics

Promensencen
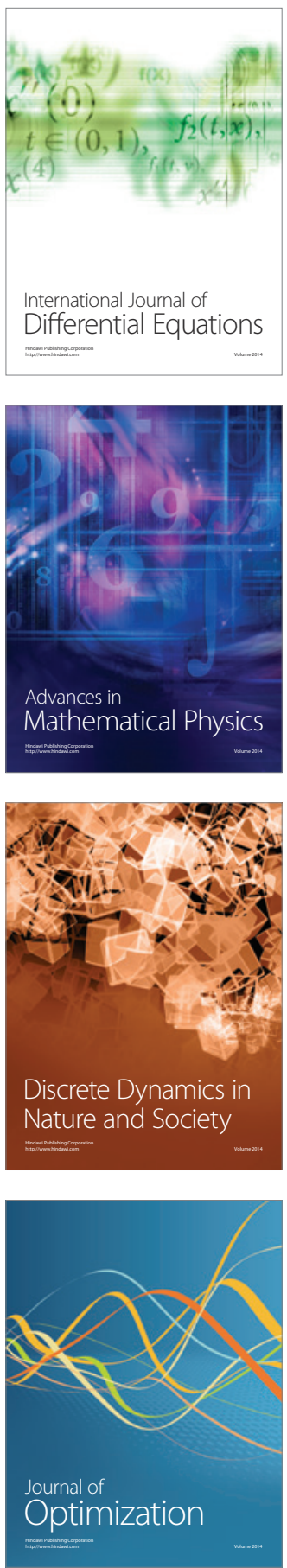